Journal of Clinical and Nursing Research

Research Article

\title{
Advances in Acupuncture and its Associated Therapies for the Prevention and Treatment of Obesity Type 2 Diabetes Mellitus
}

\author{
Kaiya Zhang ${ }^{1}$, Yuanyuan Ren ${ }^{2 *}$, Yanyan Zhou ${ }^{1}$ \\ ${ }^{1}$ Shaanxi University of Traditional Chinese Medicine, Xianyang, Shaanxi,712046; \\ ${ }^{2}$ Xi' an Hospital of Traditional Chinese Medicine, Xi'an, Shaanxi,710021;
}

Funding: Project of Xi'an Health Planning and Family Planning Commission (J201901001); Fundamental specialty of Shaanxi Provincial Administration of traditional Chinese medicine (Shi Wei Ji Fa [2018] No. 27); Natural Science Foundation of Shanxi Province of of China(2020JM-696); Xi'an Fang's Scalp Acupuncture School Inheritance Studio Project \{Shi Wei Ji Han [2018] No. 699\};

\begin{abstract}
Obesity type 2 diabetes mellitus is a common metabolic disease in clinical practice, and its prevalence is increasing rapidly with the aging of the population and changes in lifestyle. Acupuncture, as a distinctive therapy, has its unique advantages in the treatment of obesity type 2 diabetes and has an irreplaceable role in a variety of treatment methods. The author organized the literature on acupuncture and its related therapies to prevent and treat obesity type 2 diabetes in recent years and found that acupuncture and its associated therapies to prevent and treat obesity type 2 diabetes mainly include: simple acupuncture, electroacupuncture, acupoint catgut embedding therapy, auricular-plaster therapy and other treatments, all of which can safely and effectively improve clinical symptoms, acupuncture and its related therapies to treat obesity type 2 diabetes has a broad prospect, worthy of further clinical promotion.

Keywords: Obesity type 2 diabetes; Acupuncture; Acupoint catgut embedding therapy; Auricular-plaster therapy; Review
\end{abstract}

Publication date: September, 2020

Publication online: 30 September, 2020

*Corresponding author: Yuanyuan Ren, yuanyuan ren@126.com

Type 2 diabetes mellitus (T2DM) is a metabolic disorder manifested by persistently high levels of blood glucose, caused by a gradual increase in insulin resistance and a decrease in insulin secretion. It is one of the most severe epidemics of the time ${ }^{[1]}$. Obesity and T2DM are mutually exclusive risk factors, and co-morbidity dramatically increases the risk of cardiovascular disease ${ }^{[2]}$. The treatment of obese T2DM requires strict control of blood glucose and body weight, which, together with the fact that most patients have underlying diseases, dramatically limits the choice of treatment modalities. In recent years, acupuncture as a treatment of obese T2DM has been fully recognized in clinical practice; the author will summarize the current literature on acupuncture and its related therapies for the treatment of this disease, to provide a reference for clinical workers.

\section{Acupuncture therapy}

Acupuncture treatment has achieved specific efficacy in both reducing patient symptoms and improving clinical indicators, improving the quality of life of obese T2DM patients and is expected to be a green and safe treatment $[3,4]$. Dai Chenggang et al observed the clinical efficacy of acupuncture on obese T2DM patients ${ }^{[5]}$. In the control group, siglitine tablets were taken orally. In the acupuncture group, acupuncture was combined with acupuncture treatment on the basis of the control group. After eight weeks of treatment, the total 
effective rate of the acupuncture group was $90.00 \%$, significantly better than $63.33 \%$ of the control group $(\mathrm{P}<0.05)$. It has more advantages in improving FPG, $2 \mathrm{hPG}$ and $\mathrm{HbA} 1 \mathrm{c}$, reducing $\mathrm{TC}$ and TG, and improving adiponectin, Adropin and IRisin. More advantageous in terms of adiponectin, a drop in and irisin. Chen Chuanxiu ${ }^{[6]}$ compared the efficacy of acupuncture combined with pharmacological treatment of knots and pharmacological treatment of obese T2DM alone; the treatment group was treated with acupuncture combined with metformin. The control group was treated with metformin, and after three treatment sessions, both groups showed improvement in TCM clinical symptom points, obesity indexes, and glucose metabolism biochemical indexes, and the treatment group's TCM clinical symptom points, BMI, WC, FPG, and LDL and HOMA-IR were superior to the control group $(\mathrm{P}<0.05)$.

\section{Electroacupuncture}

Electroacupuncture therapy can exert the synergistic effect of acupuncture and electricity, not only replacing the medical practitioner's manipulative needle transport but also accurately grasping the amount of stimulation $^{[7]}$. Zhang $\mathrm{Na}$ et al used electroacupuncture on 203 patients with bilateral plantar and sanyinjiao, and after three months of treatment, the results showed that 203 patients were significantly active in 97 cases, effective in 82 cases, and ineffective in 24 cases, and the results showed that electroacupuncture could not only control the blood glucose level of obese T2DM patients well and protect the function of pancreatic $\beta$-cells but also reduce the obesity index of patients ${ }^{[8]}$. Xiang Qiongyao et al randomly divided 64 obese T2DM patients into a drug group and an electroacupuncture group, and the electroacupuncture group was selected from the pivot and the foot, and after three months of treatment, it was found that both treatments could reduce patients' body weight, glucose metabolism index and insulin resistance index, and electroacupuncture was more effective than drugs in reducing body weight, improving glucose metabolism and insulin resistance level ${ }^{[9]}$.

\section{Acupoint catgut embedding therapy}

Acupuncture and thread burial therapy is a combination of traditional acupuncture therapy and modern medicine, not only has the effect of ordinary acupuncture but also has the long-term impact of acupuncture "still to stay". Because of its time- consuming and straightforward method of operation, it is becoming an effective method for clinical weight loss and glucose reduction ${ }^{[10,11]}$. Studies have shown that acupuncture points can reduce pancreatic $\beta$-cell apoptosis, slow down insulin resistance and reduce body weight ${ }^{[12,13]}$. Huang Tao buried acupuncture points in the abdomen of 16 cases of obese T2DM patients ${ }^{[14]}$. After the end of treatment, the patients' body mass was reduced, and fasting blood glucose and glycosylated hemoglobin was also reduced accordingly, indicating that acupuncture point burial can not only play a role in weight loss but also control blood glucose. Wu Yuyi et al randomly divided 120 obese T2DM patients into a treatment group and a control group, and in the treatment group, 3-0 catgut was buried at the acupoint ${ }^{[15]}$. After 3 months of treatment, the relevant indicators of patients were significantly improved, and the difference was statistically significant.

\section{Auricular-plaster therapy}

The ear acupuncture points are closely related to the physiological functions and pathological changes of the internal organs, and by stimulating some response areas of the ear, the services of the corresponding internal organs can be regulated ${ }^{[16-18]}$. Fan Chunling et al randomly divided obese T2DM patients into a control group, an acupuncture group, and an earacupuncture group of 30 cases each $^{[19]}$. The acupuncture and ear group was treated with auricular patching in addition to the first two groups, and after three months of treatment, the glycated hemoglobin and body fat rates of the acupuncture and ear groups were found to be significantly lower than those before surgery, and it was concluded that auricular patching therapy could effectively control blood glucose in obese T2DM patients, while substantially improving body fat rates. Fan Chunling et al also found that acupuncture and auricular applanation therapy could significantly alleviate the clinical symptoms of patients due to metabolic disorders, with excellent efficacy ${ }^{[20]}$.

\section{Summary and outlook}

According to Chinese medicine, the onset of thirstquenching is usually caused by dysfunction of internal organs, and the pathogenesis can involve multiple internal organs such as spleen, kidney, pancreas, etc. Therefore, the treatment of obese T2DM with acupuncture is usually based on the regulation of internal organs, balancing yin and yang. 
In summary, there are many ways to treat obese T2DM with acupuncture, and acupuncture can reduce clinical symptoms and improve clinical indicators of obese T2DM patients. However, there are still the following deficiencies in the current clinical studies: (1) the reviews are mostly small sample observations, with short follow-up cycles, lacking an absolute scientific validity; (2) the diagnostic criteria and efficacy evaluation criteria vary greatly, and the control group settings are not standardized enough, lacking reproducibility; (3) the studies on the early onset of the disease and the mechanisms of the disease are few and not in-depth. Therefore, it is necessary to conduct large-sample, multicenter, randomized controlled studies, strictly develop diagnostic criteria and efficacy evaluation criteria, standardize the settings of the control group, and strengthen the reproducibility of acupuncture treatment; enhance research on the pathogenesis of the disease, explain the mechanism of acupuncture treatment, provide a reference for future clinical research, so that acupuncture treatment of obese T2DM can be better promoted.

\section{References}

[1] Zimmet, P.Z., Magliano, D.J. and Herman, W.H. et al. Diabetes: a 21 st-century challenge.[J]. The lancet. diabetes \&amp; endocrinology, 2014, 2(1): 56-64.

[2] Wang L, Gao P, Zhang M, et al. Prevalence and Ethnic Pattern of Diabetes and Prediabetes in China in 2013.[J]. JAMA, 2017, 317(24) : 2515-2523.

[3] Yang Y, Liu YX. Acupuncture and moxibustion for the treatment of obese type 2 diabetes mellitus: clinical research progress[J]. Modern Chinese Medicine, 2014, 21(3):53-56.

[4] Zhou PN, Peng PM, Wang RD. Acupuncture and moxibustion for the treatment of new-onset obesity and type 2 diabetes mellitus[J]. Acupuncture and Moxibustion Clinical Journal, 2013, 29(1): 21-23.

[5] Dei CG, Chen G, Yang JJ, et al. Effect of acupuncture combined with sitagliptin on adiponectin, a drop in and irisin in obese type 2 diabetic patients[J]. Liaoning Journal of Traditional Chinese Medicine, 2019, 10: 2172-2175.

[6] Chen YX. Effects of acupuncture and medicine on insulin resistance and biochemical metabolism in patients with type 2 diabetes mellitus and obesity[J]. Shanghai Journal of Acupuncture and Moxibustion, 2013, 32(11): 911-913.

[7] Leng QY, Han MZ, Li XH. Electroacupuncture for the treatment of diabetic peripheral neuropathy[J]. Hebei TCM, 2019, 41(1): 156-160

[8] Zhang N, Liu ZC, Xu B. Electroacupuncture treatment of T2DM with obesity in 203 cases[J]. World Science and Technology-Chinese Medicine Modernization, 2014, 16(8): 18091813.

[9] Xiang QY, Li GA, Shen XH, et al. Electroacupuncture combined with auricular acupuncture intervention in simple obesity with impaired glucose regulation: a clinical study[J]. Shanghai Journal of Traditional Chinese Medicine, 2013, 47(5): 31-33.

[10] Liu TT, Zeng JL. Progress in the application of acupuncture points buried in various clinical disciplines[J]. Clinical Journal of Chinese Medicine, 2016(11): 1636-1638.

[11] Fang S, Wang M, Zheng Y, et al. Acupuncture and Lifestyle Modification Treatment for Obesity: A Meta-Analysis[J]. The American journal of Chinese medicine,2017, 45(2): 239-254.

[12] Li Y, Shen YP. Effect of acupuncture point burial on lipocalin and its receptors in an obese rat model[J]. World Traditional Chinese Medicine, 2015, 10(11): 1752-1759.

[13] Guan ZM, Bai XH, Qu L, et al. Effect of acupuncture point burial and glucagon drink on serum TGF and IGF-1 in rats with early diabetic nephropathy[J]. Journal of Guiyang Medical College, 2015, 40(12): 1352-1355.

[14] Huang T. Observation of glycemic control of central obese diabetes mellitus by abdominal acupoint burial with traditional Chinese medicine[J]. China Acupuncture and Moxibustion, 2017, 12: 1287-1288.

[15] Wu YY, Luo QF, Wu MQ, et al. Clinical study on the treatment of obesity type 2 diabetes by acupuncture point burial[J]. Clinical Engineering, 2017, 24(10): 1379-1380.

[16] Wang XL, Lin GH, Xu N, et al. Analysis of reports of adverse reactions to acupuncture point burial[J]. Chinese Acupuncture and Moxibustion, 2020, 2: 193-196+210.

[17] Zhang W, Yang LS, Tang JX, et al. Case and analysis of rejection reaction after embedding chrome sheep intestinal thread acupuncture points[J]. China Acupuncture and Moxibustion, 2015, 35(4): 372-374.

[18] Qian L, Lou R, Huang KQ, et al. Effect of integrated ear acupuncture therapy on blood glucose in patients with type 2 diabetes mellitus[J]. Shanghai Journal of Acupuncture and Moxibustion, 2017, 36(5): 555-557.

[19] Fan CL, Ding XY, Ma YH, et al. Clinical observation of acupuncture combined with auricular pressure for the treatment of obese type 2 diabetes[J]. Shanghai Journal of Acupuncture and Moxibustion, 2019, 38(10): 1079-1082.

[20] Fan CL, Zhang Y, Zhang J, et al. Acupuncture combined with auricular acupressure for the treatment of obese type 2 diabetes in Chinese medicine[J]. Clinical Journal of Chinese Medicine, 2019, 31(3): 522-524. 